\title{
Wende, Wolfgang; Nijhuis, Steffen; Mensing-de Jong, Angela; Humann, Melanie (Hrsg.) (2020): Inclusive Urbanism. Advances in research, education and practice
}

\author{
Joachim Hartlik
}

Eingegangen: 14. Oktober 2020 - Angenommen: 13. Januar 2021 - Online veröffentlicht: 29. Januar 2021

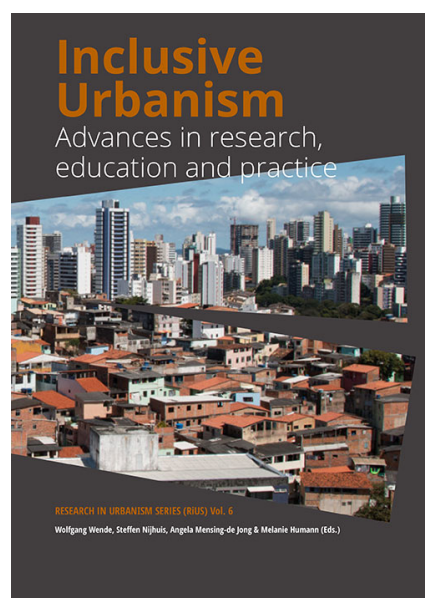

„Inclusive Urbanism“ enthält ausgewählte Beiträge einer internationalen Konferenz über „Urban Studies“ am 15. und 16. November 2018 an der Technischen Universität Dresden. Die Konferenz wurde gemeinsam von der TU Dresden, der TU Delft, dem Leibniz-Institut für ökologische Raumentwicklung (IÖR), der Tschechischen Technischen Universität Prag und der Wrocław University of Science and Technology organisiert.

Gegenwärtig steht die Gesellschaft vor großen Herausforderungen im Zusammenhang mit der fortschreitenden Urbanisierung. Mehr als die Hälfte der Weltbevölkerung lebt bereits in Städten und das rasche Wachstum der Städte scheint

Dr. Joachim Hartlik, Büro für Umweltprüfungen und Qualitätsmanagement, Kreuzkamp 5s, 31275 Lehrte, Deutschland info@hartlik.de

(c) (1) (2) ( 2021 Hartlik; licensee oekom verlag. This Open Access article is published under the Creative Commons Attribution-ShareAlike 4.0 International Licence. weiterzugehen. Im Jahr 2050 werden es laut einer Prognose der UNO rund zwei Drittel sein. Die Gemeinschaftspublikation des Leibniz-Instituts für ökologische Raumentwicklung (IÖR), der TU Dresden und dem Bereich Urbanism der TU Delft widmet sich den daraus resultierenden Fragestellungen. Im Mittelpunkt der Stadtentwicklung stehen dabei nicht mehr einzelne spektakuläre Gebäude, sondern vielmehr das soziale Miteinander in urbanen Räumen und insbesondere auch die Teilhabe ökonomisch oder sozial benachteiligter Gruppen.

Die mit der fortschreitenden Urbanisierung verbundene großräumige Umgestaltung städtisch geprägter, aber auch ländlicher Räume rückt Umweltfragen und solche der Nachhaltigkeit in den Mittelpunkt. Die Ziele der Vereinten Nationen für eine nachhaltige Entwicklung (sustainable development goals) und die „New Urban Agenda“ sowie „Habitat III“ bieten einen Rahmen für eine schnelle Stadtentwicklung, indem sie die Integration sozialer und ökologischer Aspekte in den Städtebau fördern. Eine wichtige Aufgabe des Städtebaus besteht darin, Städte inklusiv, sicher, widerstandsfähig und nachhaltig zu machen. Die Frage, wie sich Städte einerseits an die Folgen des Klimawandels anpassen und andererseits im Sinne des Klimaschutzes nachhaltiger und sozial ausgewogener entwickeln können, steht dabei über allem.

Was dies für Forschung, Bildung und Praxis in der räumlichen Planung bedeutet, sind die Kernfragen der Publikation. Das Buch konzentriert sich auf den inklusiven Urbanismus als einem wesentlichen Faktor der nachhaltigen Stadtentwicklung. Inklusivität bzw. Inklusion im Sinne des Buchtitels bedeutet, der städtischen Segregation - und als deren Folgen wirtschaftlicher Ausgrenzung, sozialer Ausgrenzung und schlechtem Zugang zu städtischen Qualitäten und ökologischen Ressourcen - entgegenzuwirken. Inklusi- 
vität ist daher stark mit städtischer Gerechtigkeit, Umweltgerechtigkeit sowie partizipatorischen Ansätzen verknüpft.

Die Fachbeiträge enthalten wichtige Beobachtungen zu den Entwicklungen von sozialökologisch-inklusiven Theorien und Praktiken auf den Gebieten der Landschaftsplanung/-architektur, des Städtebaus und der Stadtplanung aus der Perspektive von Forschung, Ausbildung und praktischen Anwendungen und sollen damit einen Überblick zum state of the art dieser neuen kooperativen Planungsansätze aufzeigen.

Weitere Betrachtungsgegenstände der Publikation sind die Untersuchung öffentlicher und grüner Räume mit ihren Möglichkeiten zu Kooperation, Integration und Partizipation sowie die Beschreibung von Fallbeispielen aus verschiedenen Teilen der Welt, die die Möglichkeiten für belastbare, integrative und nachhaltige Planungsprozesse der Stadt- und Landschaftsentwicklung demonstrieren.

Die brasilianische Wissenschaftlerin Alexandra Aguiar Pedro untersuchte beispielsweise im Dresdner Stadtteil Hellerau das interkulturelle Urban-Gardening-Projekt GolgiPark. Unter den richtigen Voraussetzungen können solche Initiativen wertvolle Integrationsarbeit leisten und Menschen soziale Teilhabe ermöglichen. Die Studie evaluierte die Leistung städtischer Gemeinschaftsgärten, um ihr Potenzial für die Umsetzung in den Slums von Sao Paulo, Brasilien, zu überprüfen. Anhand vorhandener Studien wurden bedeutende Vor- und Nachteile analysiert und in soziale, räumliche, wirtschaftliche und ökologische Faktoren eingeteilt. Zusätzlich wurden qualitative Interviews zu gesellschaftlichen und motivationalen Fragen mit Mitarbeiterinnen/Mitarbeitern eines Gemeinschaftsgartens in Dresden durchgeführt. Die Erkenntnisse nutzt die Forscherin und Stadtplanerin nun, um in mehreren Favelas in ihrer Heimatstadt São Paulo ein ähnliches Urban-GardeningProjekt aufzubauen. Die Ergebnisse verdeutlichen das Potenzial von Urban Gardening, dem räumlichen Druck durch die Schaffung von Grünflächen, die Verbesserung der Lebensmittelqualität, die Steigerung des Umweltbewusstseins und ganz allgemein durch die Gewährleistung einer höheren Lebensqualität entgegenzuwirken.

Das Thema ist nur eines von vielen weiteren forschungsund praxisbezogenen Themen in diesem Buch. Gleich mehrere Artikel beschäftigen sich mit der Partizipation der Bevölkerung an kooperativen Planungsprozessen und entwerfen alternative Szenarien zu einer Stadtentwicklung/-planung im herkömmlichen Sinn. Von einer deutschen Kleinstadt, in der die Beteiligung von Jugendlichen erprobt wird, bis zu den neuen Megastädten in Afrika, wo gemeinsame Workshops von Planerinnen/Planern und Bevölkerung für mehr Inklusion im Sinne einer gerechten Teilhabe sorgen sollen, reichen die Fallbeispiele. Sogenannte informelle Siedlungen, die nicht nur im globalen Süden in Form riesiger Slums existieren, sind heute nicht mehr nur negativ anzusehen. Vielmehr erkennen die Planenden die Chancen spontaner Urbanisierungsprozesse und suchen nach Möglichkeiten, die Menschen in ihrer Selbstorganisation zu unterstützen.

Fazit: Es handelt sich insgesamt um ein außergewöhnliches, anspruchsvolles und eindringliches Fachbuch, das Augen öffnet, Horizonte erweitert, die gewohnte Sichtweise der Stadt- und Landschaftsplanenden überschreitet und sich den aktuellen Fragen, die mit der Urbanisierung, aber auch den nicht nachlassenden Migrationsprozessen verbunden sind, in beeindruckender Weise stellt, in dem praktische Lösungswege aufgezeigt werden.

\section{Vollständige bibliographische Angaben des rezensierten Werkes:}

Wende, Wolfgang; Nijhuis, Steffen; Mensing-de Jong, Angela; Humann, Melanie (Hrsg.) (2020): Inclusive Urbanism. Advances in research, education and practice. Delft: TU Delft OPEN. = Research in Urbanism Series 6, 326 Seiten. 